\title{
Comprehensive geriatric assessment (CGA): the best integrated care approach against frailty
}

\begin{abstract}
Frailty, a complex geriatric syndrome, yields many negative impacts at Old Age. The best care approach to managing Frailty is provision of a comprehensive geriatric assessment (CGA). The essential components of a CGA should be recognised well and this integrated care must be provided to the frail older people in timely, to minimise the unfavourable consequences of Frailty.
\end{abstract}

Keywords: Frailty, comprehensive geriatric assessment, different clinical settings
Volume I Issue 4 - 2017

\author{
Moe Thaw O,' G Shorthouse, I AL Ridgeway, ${ }^{2}$ \\ JWalker ${ }^{2}$ \\ 'Department of Geriatric Medicine, Sandwell Hospital, UK \\ ${ }^{2}$ University of Warwick, UK
}

Correspondence: Moe Thaw O, Department of Geriatric Medicine, Consultant Physician and Hon., Sandwell Hospital, UK, Email moethaw.oo@nhs.net

Received: October 28, 2016 | Published: June 13, 2017

\section{Frailty and comprehensive geriatric assessment (CGA)}

Frailty is a major challenge in the older people. At old age, Frailty emerges as a result of reducing physiological reserves in the body. ${ }^{1}$ It causes many negative impacts in an older person. These impacts include recurrent unplanned admissions, prolong in-patient stay, institutionalization and high in-patient mortality. 2,3

When frailty is detected in an older person at any clinical setting, early interventions or an integrated assessment should be provided promptly $^{4}$ as frailty can increase the risks of all negative outcomes: recurrent hospitalisation, institutionalization, disability and death in old age. ${ }^{4,5}$ At this stage the main task is to determine the kind of intervention that is best for frail older people. In 2002, FiataroneSingh ${ }^{6}$ proposed that individualised resistance and balance exercises could halt the progress of frailty. ${ }^{6}$ Morley and colleagues also pointed out those managing underlying medical illnesses such as diabetes mellitus, congestive cardiac failure and anaemia is critically important in fighting against frailty. ${ }^{4}$

Asthana and colleagues suggested that testosterone replacement should be considered in frail, older males with this specific hormonal deficiency. ${ }^{7}$ Like Fiatarone-Singh's suggestion, Theou and colleagues found that individualised multi-component physical exercises of long duration (more than five months) combined with shorter-duration sessions (three times per week, 30-45 minutes per session) could help frail, older people gain benefits in terms of physical activities. ${ }^{8}$ These benefits include increased walking speed, stair climbing, balance, chair stand and decreased fear of falls. ${ }^{8}$

In addition to the assessment of physical activities, nutrition is critical in the management of frailty. ${ }^{9}$ Nutritional deficiencies, particularly vitamin D deficiency should be detected and treated appropriately in order to prevent and control frailty.,9 Vitamin D with a calcium supplement could improve physical performance and limb muscle strength in older people with this deficiency., ${ }^{4,9}$ Tieland et al. ${ }^{10}$ mentioned that overall physical activities significantly improved in the group of frail older participants who received $15 \mathrm{~g}$ protein supplements daily, compared with the placebo group in their randomised double-blind placebo-controlled trial. ${ }^{10}$
A Cochrane Database study in 2002 showed us that regular protein supplementation in an appropriate amount could reduce inpatient stay (up to 3.4 days), mortality and gain body weight in older, frail patients with malnutrition. ${ }^{4,9}$ The INTERCOM trial, a Dutch interdisciplinary community-based study, found that supplementation of nutrients as required provided weight gain, increased walking distance by six minutes and reduced the frequency of hospitalisation. ${ }^{4,11}$

For hospitalized frail, older people comprehensive geriatric assessment (CGA) is the best intervention to provide integrated multi-disciplinary care. ${ }^{9}$ A randomized controlled trial, regarding multi-disciplinary treatment of mortality and nursing home admissions after hip fractures, concluded that CGA with longterm physical rehabilitation decreased post-hip fractures mortality, institutionalization and increased independent level of activities of daily living in older frail people. ${ }^{9,12}$ A meta-analysis of CGA in elderly inpatients concluded that elderly patients who received CGA in hospitals were likely to survive well or deteriorate less than those who did not receive CGA. ${ }^{13}$

\section{Characteristics of CGA}

\section{Aim of the assessment}

The aim of CGAs is to maintain and promote the overall good health of older people. ${ }^{14}$

\section{Indications for the CGA}

In general, age is an absolute indication for this assessment. People aged 75 years and over should receive a CGA. However, age limits should be varied depending on the needs of the local older population. The other indications for a CGA include underlying medical comorbidities, cognitive impairments, falls and poor mobility, weight loss and nutritional deficiencies and complex social circumstances including at-risk vulnerable adults. For frail older people it is indicated as an essential requirement. ${ }^{14}$

\section{CGA assessment team}

A CGA is a multi-disciplinary team-based assessment requiring different healthcare professionals with different expertise: physicians, physiotherapists, occupational therapists, a mental health team, 
dieticians and social workers, should be actively involved in this assessment. ${ }^{14}$

\section{Components of CGA}

Its components are divided into two categories:

\section{Essential components}

a. Managing acute illnesses or chronic medical conditions

b. Assessing mobility and function capacities

c. Assessing social supports

d. Assessing memory and cognitive function

e. Assessing nutritional status ${ }^{14}$

\section{Additional components}

a. Reviewing poly-pharmacy

b. Assessing visual acuity

c. Assessing hearing

d. Assessing dentition

e. Assessing bowel and urinary incontinence

f. Assessing sexual functions (if indicated)

g. Assessing and preventing any kind of abuse (physical, psychological, social and financial)

h. Assessing financial concerns

i. Establishing appropriate long-term care plans.14

\section{Places where CGA should be conducted}

Based on current evidence, the CGA should be undertaken in secondary care or hospital settings, primary care settings, outpatient clinic settings and during domiciliary visits. ${ }^{14}$

\section{Secondary care or hospital settings}

Acute geriatric care including CGAs is vital for frail older patients in hospitals. A meta-analysis found that inpatient CGA provided positive overall outcomes for older patients on discharge, increased functional capacities of patients, decreased institutionalisation and inpatient mortality. ${ }^{15}$

\section{Primary care settings}

Though the key principles and components of a CGA performed in primary care settings are the same as those in secondary care there are some unique characteristics of the assessment in general practice. Primary care teams led by general practitioners provide followup assessment, optimise chronic medical conditions, rationalise polypharmacy and continue long-term rehabilitation assessment for these older patients post-discharge. ${ }^{14}$ These care activities are conducted through the CGA. Communication with a secondary care team, referral to outpatient specialty services, social services and community mental health services are also undertaken as a part of a CGA. ${ }^{14}$ Applying this integrated approach reduces and prevents emergency hospital admissions as well as re-admissions of older people. $^{16}$

\section{Outpatient clinic settings}

According to a randomised trial, Geriatric Resources for Assessment and Care of Elders (GRACE), interventions among low income elderly people who received a CGA in outpatient clinics had better overall health quality and less emergency admissions than those who did not receive interventions. ${ }^{17}$

\section{Domiciliary visit}

Home-based CGA is mainly aimed at providing preventive care rather than curative or rehabilitative services. ${ }^{14} \mathrm{~A}$ meta-analysis of various trials regarding home-based CGA found that this assessment could reduce functional decline and overall mortality, ${ }^{18}$ but it could not decrease the chance of institutionalisation. ${ }^{18}$

\section{Conclusion}

A Comprehensive Geriatric Assessment (CGA) should be applied proactively in all clinical settings for frail older people. It is the best crisis-intervention as well as preventive measure in managing frail older people with complex co-morbidities.

\section{Acknowledgements}

None.

\section{Conflict of interest}

Authors declare there is no conflict of interest in publishing the article.

\section{References}

1. Clegg A, Young J, Iliffe S, et al. Frailty in elderly people. Lancet. 2013;381(9868):752-762

2. Fried LP, Tangen CM, Walston J, et al. Frailty in older adults: evidence for a phenotype. J Gerontol A Biol Sci Med Sci. 2001;56(3):146-156.

3. Rockwood K, Mitnitski A, Song X, et al. Long-term risks of death and institutionalization of elderly people in relation to deficit accumulation at age 70. J Am Geriatr Soc. 2006;54(6):975-979.

4. Morley J, Matthew H, Rolland $\mathrm{Y}$, et al. Frailty. Med Clin N Am. 2006;90(5):836-847.

5. Shinkai S, Watanabe N, Yoshida H, et al. Research on screening for frailty: development of the "Kaigo-Yobo Checklist". Nihon Koshu Eisei Zasshi. 2010;57(5):345-354.

6. Fiatarone $\mathrm{S}$. Exercise in the oldest old: sorne new insights and unanswered questions. J Am Genatr Soc. 2002;50(12):2089-2091.

7. Asthana $\mathrm{S}$, Shasin $\mathrm{S}$, Butler RN, et al. Masculine vitality: pros and cons of testoslerone in treating the andropause. J Gerontol A Biol Sci Med Sci. 2004;59(5):461-465.

8. Theou O, Stathokostas L, Roland KP, et al. The effectiveness of exercise interventions for the management of frailty: a systematic review. J Aging Res. 2011;4:569-594.

9. Abellan van Kan G, Rolland YM, Morley JE. Frailty: toward a clinical definition. J Am Med Dir Assoc. 2008;9(2):71-72.

10. Tieland M, van de Rest O, Dirks ML, et al. Protein supplementation improves physical performance in frail elderly people: a randomized, double-blind, placebo-controlled trial. $J$ Am Med Dir Assoc. 2012;13(8):720-726 
11. Van Wetering CR, van Nooten FE, Mol SJ, et al. Systemic impairment in relation to disease burden in patients with moderate COPD eligible for a lifestyle program. Findings from the INTERCOM trial. Int J Chron Obstruct Pulmon Dis. 2008;3(3):443-451.

12. Singh NA, Quine S, Clemson LM, et al. Effects of high-intensity progressive resistance training and targeted multidisciplinary treatment of frailty on mortality and nursing home admissions after hip fracture: a randomized controlled trial. J Am Med Dir Assoc. 2012;13(1):24-30.

13. Ellis G, Whitehead MA, Robinson D. Comprehensive geriatric assessment for older adults admitted to hospital: meta-analysis of randomised controlled trials. BMJ. 2011;343:d6553.

14. http://www.uptodate.com/contents/comprehensive-geriatric-assessment
15. Bachmann S, Finger C, Huss A, et al. Inpatient rehabilitation specifically designed for geriatric patients: systematic review and meta-analysis of randomised controlled trials. BMJ. 2010;340:c1718.

16. Hesselink G, Schoonhoven L, Barach $\mathrm{P}$, et al. Improving patient handovers from hospital to primary care: a systematic review. Ann Intern Med. 2012;157(6):417-428.

17. Counsell SR, Callahan CM, Clark DO, et al. Geriatric care management for low-income seniors: a randomized controlled trial. JAMA. 2007;298(22):2623-2633.

18. Huss A, Stuck AE, Rubenstein LZ, et al. Multidimensional preventive home visit programs for community-dwelling older adults: a systematic review and meta-analysis of randomized controlled trials. J Gerontol A Biol Sci Med Sci. 2008;63(3):298-307. 